\title{
Memory Engram Studies and the Brain-computer Interface: The Possibility of Visibly Artificial Memory Retrieval
}

\author{
$\mathrm{KeFu} \mathrm{Mu}$ \\ Development of computer science \& engineering, Sichuan University of Science \& Engineering, China \\ Corresponding author. Email:raysonmu@163.com
}

\begin{abstract}
Advances in the study of memory engrams are exciting in the development of neuroscience. Through optogenetic techniques, scientists have succeeded in awakening dormant memories in mice, and although the experiments were limited to animals, this has provided a theoretical and practical basis for future artificial memory awakening techniques. The development of BCI is equally noteworthy, and it attracts the attention of many in the scientific community gradually. In recent visual BCI studies, researchers have decoded the state of the human brain when visual stimuli are generated and reconstructed it into images by using a new neurofeedback model, which also makes it possible to conduct future visualization studies of memory. After summarizing the developments on memory engram and BCI studies and combining them, it was found that visibly artificial memory retrieval techniques may be realized in the future. Before that, however, more research on brain signaling is needed, and the pharmacological and technical difficulties in human clinical trials remain a challenge for scientists.
\end{abstract}

Keywords: memory retrieval, engram, brain-computer interface, visual BC system, neuroscience

\section{INTRODUCTION}

Memory is fundamental to the intelligence humans possess, because it defines who we are. Without memory storage people would not have experience, and without memory integration people would not be able to correlate things. Engram is the basic unit of memory, and the research on it runs through the exploration of the nature of the brain which is the most sophisticated operating system of human. Brain-computer interface (BCI) is a system that enables real-time communication between the brain and external devices by converting EEG and neuroelectrical signals. The development and application of memory engram research requires the cooperation of multiple disciplines such as biochemistry, informatics, and engineering, and the practical application of the discoveries made in animals to human-based clinical trials through brain-computer interfaces (BCI). The deeper development of brain-computer interface (BCI) also relies on our further research on the process of encoding, storing and extracting information from engrams in the brain.

This paper attempts to summarize the development of memory engram research and $\mathrm{BCI}$ to find some exciting ways of research brought by combining them, and to explore the possibilities of future visibly artificial memory retrieval techniques, related technical shortcomings, and future research directions. The implementation of this technique will be of great help for researchers to conduct more in-depth research on the essence of memory engram, and it has potential applications and implications in rehabilitation, treatment, criminal investigation, artificial intelligence, neuroscience and other fields. However, with the development and application of it, there are still many problems that need to be broken through, and there are also many operational problems and ethical challenges waiting to be explored.

\section{ENGRAM RESEARCH STATUS}

\subsection{History and development}

People have been exploring the nature of memory for thousands of years, starting with Aristotle's "On Memory" in 350 B.C [1]. It was not until 1905 that Semon first referred to the traces of memory formed in cells as "engrams" and suggested that engrams are 
formed by a group of neurons that undergo chemical or physical changes [2][3]. Even so, the exploration of engrams was slow for the next 100 years because of the lack of technological means. In the last decade or so, some breakthroughs in the study of engrams have been made thanks to the creation of optogenetic techniques in the Diesseroth lab at Stanford University in 2007. In 2012, the team of Susumu Tonegawa in MIT used optogenetic techniques to artificially awaken memory in mice successfully [4], confirming the existence of engrams, and then they discovered the possibility of artificially creating false memories during the memory "reconsolidation" window through experiments [5].

The development of engram research is primarily driven by the need of the treatment of neurological disorders, whether it is amnesia [6], PTSD (post-traumatic stress disorder) [7][8] or Alzheimer's disease, its symptoms are related to the storage, encoding and extraction of memories in their nervous system. According to current research, artificial activation of silent engram cells may be able to treat pre-Alzheimer's disease [9], and interference with the "reconsolidation" process of memory engrams may be able to treat PTSD patients with exposure therapy.

Based on the current state of the development of society, the aging population and the increasing number of people who suffer from neurological diseases, many patients as well as medical institutions are gradually realizing the importance and urgency of research on human memory mechanisms. Due to the lack of safe pharmacological and technical operations, most of the current experiments are based on organisms such as rats and monkeys, and relevant clinical trials have not yet been able to be conducted on humans without injury. Further development depends on more relevant technological advances [10].

\subsection{Experiment of memory expression inducing}

The research team of Susumu Tonegawa and Roy conducted an experiment that attempted to mimic the natural memory retrieval process by reactivating engram cells through optogenetic stimulation.

They bred a c-foss-tTA transgenic mouse by transgenic techniques, injected it with AAV9-TRE-ChR2-EYFP, and implanted it with fiber optics targeting DG [11]. The researchers labeled ChR2[12][13]-expressing neurons that were activated in the DG during fear learning in the mice. Later, they reactivated these neurons by light stimulation in different contexts. And it was found that in the same context associated with fear memory, mice showed strong freeze upon light stimulation which is similar to the response during fear learning, suggesting that light evokes fear memory in mice. Activation of labeled cells in a context unrelated to fear does not elicit freeze in mice, suggesting that light-evoked fear memory is context-specific.

The findings, although limited by the specific natural stimuli (i.e., the context associated with the fear memory), also provide strong evidence for the idea of artificial memory arousal.

\section{BCI RESEARCH STATUS}

\subsection{History and development}

In the 1970s, the University of California, Los Angeles (UCLA) conducted an animal experiment to develop a direct communication pathway between an external device and the brain, and this was the beginning of BCI research [14]. By 1969, scientists had conducted the first trials of BCI development in animals. The real BCI trials for humans were not conducted until the 1990s due to technical limitations. Over the past few decades, BCI research has gradually gained the attention of the scientific community, and in 2000, Jonathan Wolper defined the BCI system in its entirety for the first time [15]. In recent years, there have also been significant breakthroughs in BCI research. In 2019, a team of researchers from Russia developed a new closed-loop BCI system through artificial neural network and EEG technology, and it is capable of reconstructing images observed or imagined by a person based on brainwave parameters. Then in 2021, researchers from Stanford University developed an intrinsic BCI system that decodes neural activity in the motor cortex of the brain and translates a person's imaginary "handwriting" into text [16].

The need for BCI development comes primarily from people with disabilities, and scientists have been working to use BCI systems to control robotic exoskeletons to offer paraplegics the possibility of regaining movement. For patients with sensory loss, researchers are also trying to restore vision, hearing, and touch through BCI systems. Although current research has largely achieved the control of external machinery (e.g., intelligent wheelchairs, robotic arms, and quadcopters), and initial exploration of hybrid BCI systems (e.g., V-BCI) has been attempted (especially for rehabilitation of patients with neurological disorders) [17]. However, research on BCI is still at an early stage, and its further development also depends on data analysis in biomedicine (e.g., brain signals, etc.) and advances in AI algorithms.

\subsection{Experiment of brain images reconstructing}

A team of researchers from the Russian company Neurobotics and the Moscow Institute of Physics and Technology (MIPT) have developed a hypothesis that different brain states are generated when people observe different categories of visual stimuli, and that it may be 
possible to decode them from non-invasive electroencephalography (EEG[18]) recordings.

They developed a new closed-loop asynchronous BCI paradigm with an adaptive, modifiable set of mental states and native feedback to conduct this experiment. In the experiment, the researchers asked healthy subjects to watch a 21-minute video synthesized from 117 random video clips of five types: abstract graphics, waterfalls, human faces, mechanical devices, and first-person extreme sports. Analysis of the EEG data from the experiment revealed that each type of video stimulus produced different brain waves. The researchers then developed a visual neural feedback model based on deep learning, and mapped the 20-dimensional EEG Feature vectors obtained after dimensionality reduction into the latent space of pre-trained Image Autoencoder through EEG Feature Mapper, and then the images were reconstructed by Image Decoder [19]. After the final images were generated, they found that about $90 \%$ of the reconstructed images could be recognized and classified after testing [20].

This BCI system is non-invasive and does not require electrodes to be implanted in the brain. Its ability to reconstruct brain images in real time and visually modify brain states through visual patterns provides a solid foundation for future memory visualization studies.

\section{CURRENT RESEARCH LIMITATIONS AND FUTURE DEVELOPMENT}

Currently, artificial induction of memory expression has been achieved in mice by optogenetic techniques. This technology apparently does not support harmless clinical experiments on humans due to safety issues. The challenge for scientists is to find a new technique to induce this process of engram cell arousal. The new technique, however, may be found in our research on brain signaling and the future development and application of BCI non-invasive techniques [21]. Researchers may need to understand how the brain sends out the command to retrieve memory, and to identify how this signal is received by the engram cells and gives the corresponding feedback, which requires neuroscientists to study the mechanism behind it more deeply. BCI's non-invasive technology can help us visualize and manipulate engrams more intuitively and apply the research to humans, but its development will also require new electrode designs to accommodate electronic interfaces with large numbers of neurons, and develop more powerful computers for the signal processing [22]. In the future, an artificial memory retrieval method that may be realized is to simulate the brain instruction signal of a person's autonomous memory retrieval process through a computer, and transmit it to the brain in the form of a brain-computer interface, thereby actively activating engram cells to artificially induce the bevavior of memory retrieval. The development of the visual BCI system may also allow people to visualize the retrieved memory fragments, thus studying and analyzing memory more intuitively.

This visual artificial memory retrieval technique may be a good support for researchers in memory research to find the carriers of different types of memories that they can identify and classify the memory functions of different neurons by comparing the relationship between the memory fragment images and the active neurons in that context.

In medicine, this technology might be used for exposure therapy or specific artificial memory interventions for PTSD patients, where the patient's brain is connected to the computer through non-invasive BCI to artificially awaken his or her associated fear memory, which will be more intuitive in helping medical personnel to guide the patient to re-enter this memory scenario in a safe state, to re-perceive and challenge it. In the future, it may even be possible to modify or suppress the memory by artificially intervening in the "reconsolidation" process of the patient's memory engram, and the feasibility of this technology has been initially demonstrated in experiments that manipulate hippocampal engram cells to create false memories.

In criminal investigation cases, investigators can combine this technology to find the specific engram cells activated by this cue when reliving the crime scene for the suspect, then artificially awaken their associated memories and build them into images to reconstruct the scene and assist criminal investigators in solving the case [23].

On this basis, with more in-depth research on memory and the development of BCI systems, many scenarios in science fiction literature may become reality, using the human eyes as the highest definition camera, turning all the things and scenes seen into memories stored in the brain, so that people can play back memories just like playing back videos. Although the future is promising, it also raises some ethical issues.

\section{ETHICAL AND OPERATIONAL CONSIDERATIONS}

Neuroscience may still be at an early stage in the study of memory, and most of the experiments are still on animals. As research progresses, more and more experimental results will be validated in humans and eventually applied to human society to improve the state of human life and well-being. In this regard, while rigorous scientific experiments are important, people must not lose sight of the ethical issue that these human clinical experiments and their future applications 
involve the protection of memory, the deepest level of human privacy [24].

The same ethical issue exists for the future development of BCI, such as the gradual development of visual BCI technology that can support us to visually observe or adjust the memory state and emotional state of human brain, and the future wireless BCI technology that can implant BCI into human brain to achieve wireless brain-computer communication. All these developments have issues of adaptability, security, confidentiality and legality when they are applied to human beings [25][26].

Therefore, for the development and application of visibly artificial memory retrieval technology, people should pay more attention to some operational issues and ethical challenges.

Operational considerations:

- Evaluate the clinical trial protocol strictly and obtain the approval of the ethics committee.

- Provide adequate information to the subject or user about the operation, and then provide the most comprehensive risk prediction as possible, and obtain their permission.

- Conduct physical fitness surveys of subjects before conducting experiments.

- Standardize the operation of clinical experiments on humans, and conduct safety checks and training on the operation of relevant equipment.

Ethical considerations :

- Respect the will of the subject and protect the privacy information.

Undue influence of commercial interests on the operation and application of the technology.

- When using the technique, it may lead to excessive arousal of memory or deviation from the target, which may have unwanted effects on humans.

The future application of this technology, as it relates to human memory, may be able to help people eliminate their fear of certain things in the past, but it may also change someone's character or beliefs as a result; it may be able to prompt people to face a more realistic past and reconnect with themselves, but it may also lead to the collapse of their beliefs by destroying the idealized self that was created for self-protection[27]. Therefore, while developing and exploring it, people may need to consider its implications in advance and address the related operational and ethical issues. If this can be achieved, the future will be of great benefit not only for the development of this technology, but also for patients with memory system-related disorders who will no longer have to worry about receiving treatment.

\section{CONCLUSION}

Overall, the purpose of memory engram research is to better analyze human brain intelligence, and its research has a considerable impact on many fields such as medical, education, technology and social security, for example the treatment of memory deficit-related diseases, guided education for brain intelligence development, and intelligent development of AI, while its biggest limitation lies in its technical shortcomings. BCI research is dedicated to creating more effective Brain-computer communication bridge, and the development of its non-invasive technology has opened up more possibilities for clinical research on human memory, whether in terms of safety, convenience, or intuitiveness.

Current research on the memory engram arousal process and visual BCI system has provided convincing evidence for the implementation of visibly artificial memory retrieval techniques. For further development, we still need to understand the mechanisms behind memory retrieval in the brain and to bring the current findings to clinical trials in humans with the help of more advanced technologies (e.g., new non-invasive BCI systems). Visibly artificial memory retrieval technology has great potential for application, from clinical applications to criminal investigations, and further research by scientists and more reliable experimental support are needed for broader applications in the future.

\section{REFERENCES}

[1] Beare JI. (2010 Apr). On memory and reminiscence Aristotle (ca. 350 b.c.). Ann Neurosci. 17(2):87-91.

[2] Schacter DL, Stranger Behind the Engram: Theories of Memory and the Psychology of Science (Erlbaum Associates, 1982).

[3] Schacter DL, Eich JE, Tulving E. (1978). Richard Semon's theory of memory. J. Verbal Learn. Verbal Behav 17, 721-743.

[4] Liu X et al. (2012). Optogenetic stimulation of a hippocampal engram activates fear memory recall. Nature 484, 381-385 .

[5] Ramirez S, Liu X, Lin PA, Suh J, Pignatelli M, Redondo RL, Ryan TJ, Tonegawa S. (2013 Jul 26). Creating a false memory in the hippocampus. Science. 341(6144):387-91.

[6] Roy DS, Muralidhar S, Smith LM, Tonegawa S. (2017). Silent memory engrams as the basis for retrograde amnesia. Proc. Natl. Acad. Sci. U.S.A 114, E9972-E9979

[7] Zhang X, Kim J, Tonegawa S. (2020 Mar 18). Amygdala Reward Neurons Form and Store Fear 
Extinction Memory. Neuron. 105(6):1077-1093.e7.

[8] Maddox SA, Hartmann J, Ross RA, Ressler KJ. (2019 Apr 3). Deconstructing the Gestalt: Mechanisms of Fear, Threat, and Trauma Memory Encoding. Neuron. 102(1):60-74.

[9] Roy DS, Arons A, Mitchell TI, Pignatelli M, Ryan TJ, Tonegawa S. (2016 Mar 24). Memory retrieval by activating engram cells in mouse models of early Alzheimer's disease. Nature. 531(7595):508-12.

[10] Josselyn SA, Tonegawa S. (2020 Jan 3). Memory engrams: Recalling the past and imagining the future. Science. 367(6473):eaaw4325.

[11] Reijmers LG, Perkins BL, Matsuo N, Mayford M. (2007). Localization of a stable neural correlate of associative memory. Science. 317:1230-1233.

[12] Boyden ES, et al. (2005). Millisecond-timescale, genetically targeted optical control of neural activity. Nat Neurosci. 8:1263-1268.

[13] Tye KM, et al. (2011). Amygdala circuitry mediating reversible and bidirectional control of anxiety. Nature. 471:358-362.

[14] Kübler A. (2019). The history of BCI: From a vision for the future to real support for personhood in people with locked-in syndrome. Neuroethics. 13:163-180.

[15] Bamdad M., Zarshenas H., Auais M.A. (2015). Application of BCI systems in neurorehabilitation: A scoping review. Disabil. Rehabil. Assist. Technol. 10:355-364.

[16] Willett FR, Avansino DT, Hochberg LR, Henderson JM, Shenoy KV. (2021 May). High-performance brain-to-text communication via handwriting. Nature. 593(7858):249-254.

[17] Kawala-Sterniuk A, Browarska N, Al-Bakri A, et al. (2021). Summary of over Fifty Years with Brain-Computer Interfaces-A Review. Brain Sci. 11(1):43.

[18] Mensen A, Marshall W, Tononi G. (2017). EEG Differentiation Analysis and Stimulus Set Meaningfulness. Front Psychol; 8:1748.

[19] Simonyan, K., Zisserman, A. (2014). Very Deep Convolutional Networks for Large-Scale Image Recognition.Computer Science, 2014.

[20] Grigory Rashkov, Anatoly Bobe, Dmitry Fastovets, Maria Komarova. (2019). Natural image reconstruction from brain waves: a novel visual BCI system with native feedback. bioRxiv 787101 .

[21] Burke JF, Merkow MB, Jacobs J, Kahana MJ,
Zaghloul KA. (2015 Jan 20). Brain computer interface to enhance episodic memory in human participants. Front Hum Neurosci. 8:1055.

[22] Mridha MF, Das SC, Kabir MM, Lima AA, Islam MR, Watanobe Y. (2021 Aug 26).Brain-Computer Interface: Advancement and Challenges. Sensors (Basel). 21(17):5746.

[23] Meegan DV. (2008 Jan). Neuroimaging techniques for memory detection: scientific, ethical, and legal issues. Am J Bioeth. 8(1):9-20.

[24] Cabrera LY, Elger BS. (2016 Mar). Memory Interventions in the Criminal Justice System: Some Practical Ethical Considerations. $\mathrm{J}$ Bioeth Inq. 13(1):95-103.

[25] Steinert S, Friedrich O. (2020 Feb). Wired Emotions: Ethical Issues of Affective Brain-Computer Interfaces. Sci Eng Ethics. 26(1):351-367.

[26] Burwell S, Sample M, Racine E. (2017 Nov 9). Ethical aspects of brain computer interfaces: a scoping review. BMC Med Ethics. 18(1):60.

[27] Tan SZK, Lim LW. (2020 Sep 18). A practical approach to the ethical use of memory modulating technologies. BMC Med Ethics. 21(1):89. 